\title{
Retrospective evaluation of cases of CNS toxoplasmosis in patients with AIDS hospitalized in the Department of Hospital for Infectious Diseases, Warsaw
}

\author{
MA Miarka*, J Gizinska, D Latarska-Smuga and RB Podlasin
}

Address: Regional Hospital for Infectious Diseases, Warsaw, Poland

* Corresponding author

\author{
from Ninth International Congress on Drug Therapy in HIV Infection \\ Glasgow, UK. 9-13 November 2008 \\ Published: 10 November 2008 \\ Journal of the International AIDS Society 2008, I I (SuppI I):P258 doi:I0.II86/I758-2652-I I-SI-P258
}

This abstract is available from: http://www.jiasociety.org/content/I I/SI/P258

(C) 2008 Miarka et al; licensee BioMed Central Ltd.

\section{Purpose of the study}

The aim of the study was to characterize retrospectively the group of patients (pts) with central nervous system toxoplasmosis and AIDS.

\section{Methods}

Data were collected according to basic pts' profile, including immunological status, ARV treatment, clinical presentation of disease, treatment pattern and outcome: clinical state and neuro-imaging results were taken into consideration mainly. Autopsy examination, if performed, was evaluated as well.

\section{Summary of results}

The examined group consisted of 40 pts hospitalized in 4th Department of Regional Hospital for Infectious Diseases in Warsaw, between 1994 and 2007; 33 men and 7 women were included. Age ranged from 24 to 49 (average 34 ) years. $73 \%$ of patients had severe immunological deficit (CD4 $+<100$ cells/mm3) when CNS toxoplasmosis was diagnosed. Focal neurological deficiency, fever and disturbances of attention and concentration were the most frequent clinical signs of the disease (respectively, $71 \%, 68 \%, 65 \%)$. Neuro-imaging revealed single (56\% in CT) or multiple (70.6\% in MRI) typical focal lesion/s with peripheral enhancement with contrast (35\% in CT, $77 \%$ in MRI) and brain edema (63\% in CT and 65\% in MRI) in most cases. Majority of pts (63\%) were treated with sulfadiazine, pyrimetamine and folinic acid. In 14/27 (52\%) pts treated with sulfadiazine, adverse events complicated therapy: allergic reaction $64 \%$, nephrotoxicity $21 \%$, neutropenia $7 \%$. Complete recovery was achieved in $38 \%$ of pts; $18 \%$ pts died, the rest had some neurological complications as remnant: epilepsy $18 \%$, hemiparesis $13 \%$, cerebellar ataxia $5 \%$. In the group of pts that died, toxoplasmosis of CNS often co-existed with other neuropathology as CMV infection of CNS $(42 \%)$ or HIV encephalitis (34\%) which were revealed in autopsy examinations. Most of pts that died of CNS toxoplasmosis $(57,9 \%)$ did not receive ARV treatment, in opposition to all survivors. The secondary prophylaxis of CNS toxoplasmosis was broken by the relapse of the disease in two (9\%) cases. Six cases of CNS toxoplasmosis were developed despite the primary prophylaxis.

\section{Conclusion}

Most of our pts with CNS toxoplasmosis had severe immunodeficiency. Pts in our group presented various clinical signs of the disease. Neuro-imaging of CNS toxoplasmosis revealed mainly single in CT but multiple in MRI focal lesions. Therapy with sulfadiazine was often associated with side-effects. The prophylaxis of CNS toxoplasmosis can be insufficient in some cases. 